\title{
On a class of quartic Diophantine equations of at least five variables
}

\author{
Hamid Reza Abdolmalki ${ }^{1}$ and Farzali Izadi ${ }^{2}$ \\ ${ }^{1}$ Department of Mathematics, Faculty of Science, \\ Azarbaijan Shahid Madani University, Tabriz 53751-71379, Iran \\ e-mail: hamid.abdolmalki@gmail.com \\ ${ }^{2}$ Department of Mathematics, Faculty of Science, \\ Urmia University, Urmia 165-57153, Iran \\ e-mail: f.izadieurmia.ac.ir
}

Received: 12 January 2018

Revised: 23 August 2018

Accepted: 6 September 2018

\begin{abstract}
In this paper, elliptic curves theory is used for solving the quartic Diophantine equation $X^{4}+Y^{4}=2 U^{4}+\sum_{i=1}^{n} T_{i} U_{i}^{4}$, where $n \geq 1$, and $T_{i}$, are rational numbers. We try to transform this quartic to a cubic elliptic curve of positive rank, then get infinitely many integer solutions for the aforementioned Diophantine equation. We solve the above Diophantine equation for some values of $n, T_{i}$, and obtain infinitely many nontrivial integer solutions for each case. We show among the other things that some numbers can be written as sums of some biquadrates in two different ways with different coefficients.
\end{abstract}

Keywords: Quartic Diophantine equations, Biquadrates, Elliptic curves, Rank.

2010 Mathematics Subject Classification: 11D45, 11D72, 11D25, 11G05, 14 H52.

\section{Introduction}

The quartic Diophantine equation (DE)

$$
x^{4}+y^{4}=2 z^{4}+2 k w^{4}
$$

has already been studied by some authors. Four cases $k=1, k=6 r+1, k=-8$, and $k=2 n^{2}$, where $n$ is a congruent number, has been studied in [1-6]. 
In this paper, we are interested in the study of the DE:

$$
X^{4}+Y^{4}=2 U^{4}+\sum_{i=1}^{n} T_{i} U_{i}^{4}
$$

where $n \geq 1$, and $T_{i}$, are rational numbers. This also solves the DE (1) for some values of $k$, which is discussed in Example 1.

We conclude this introduction with a standard fact which is needed in this paper (see [8], page $37)$.

Lemma 1.1. Let $K$ be a field of characteristic not equal to 2. Consider the equation

$$
v^{2}=a u^{4}+b u^{3}+c u^{2}+d u+q^{2}
$$

with $a, b, c, d \in K$. Let

$$
x=\frac{2 q(v+q)+d u}{u^{2}}, y=\frac{4 q^{2}(v+q)+2 q\left(d u+c u^{2}\right)-\left(\frac{d^{2} u^{2}}{2 q}\right)}{u^{3}} .
$$

Define $a_{1}=\frac{d}{q}, a_{2}=c-\left(\frac{d^{2}}{4 q^{2}}\right), a_{3}=2 q b, a_{4}=-4 q^{2} a, a_{6}=a_{2} a_{4}$. Then $y^{2}+a_{1} x y+a_{3} y=$ $x^{3}+a_{2} x^{2}+a_{4} x+a_{6}$.

The inverse transformation is

$$
u=\frac{2 q(x+c)-\left(\frac{d^{2}}{2 q}\right)}{y}, v=-q+\frac{u(u x-d)}{2 q} .
$$

The point $(u, v)=(0, q)$ corresponds to the point $(x, y)=\infty$ and $(u, v)=(0,-q)$ corresponds to $(x, y)=\left(-a_{2}, a_{1} a_{2}-a_{3}\right)$.

\section{Main results}

We prove the following main theorem:

Theorem 2.1. Consider the $D E(2)$, where $n \geq 1$, and $T_{i}$, are rational numbers. There exists a corresponding quartic elliptic curve in the form $V^{2}=a t^{4}+b$, in which the coefficients $a$ and $b$ are all functions of $T_{i}$ and the other rational parameters to be found later. If this elliptic curve has positive rank, depending on the values of $T_{i}$ and the other rational parameters, the DE (2) has infinitely many integer solutions.

Proof. Firstly, it is clear that if we find rational solutions of the DE (2), then by canceling the denominators of $X, Y, U, U_{i}$, and by multiplying both sides of the DE (2) by the appropriate number $M$, we may obtain integer solutions for the DE (2).

Let $X=U+t, Y=U-t$, where all variables are rational numbers. By substituting these variables in the DE (2), and some simplifications, we get:

$$
12 U^{2} t^{2}=-2 t^{4}+\sum_{i=1}^{n} T_{i} U_{i}^{4}
$$


Let $U t=V$. Then we obtain:

$$
V^{2}=-\frac{t^{4}}{6}+\frac{\sum_{i=1}^{n} T_{i} U_{i}^{4}}{12}
$$

We see that if

$$
Q:=\frac{\sum_{i=1}^{n} T_{i} U_{i}^{4}}{12}
$$

is to be square, say $q^{2}$ (This is possible by choosing appropriate values for $U_{i}$, and $T_{i}$.), we may use from the Lemma 1.1 and transform the quartic elliptic curve (4) to an elliptic curve of the form $y^{2}+a_{1} x y+a_{3} y=x^{3}+a_{2} x^{2}+a_{4} x+a_{6}$, where $a_{i} \in \mathbb{Q}$.

Let

$$
q^{2}=\frac{\sum_{i=1}^{n} T_{i} U_{i}^{4}}{12}
$$

Then with the inverse transformation $V=-q+\frac{t^{2} X^{\prime}}{2 q}$ and $t=\frac{2 q X^{\prime}}{Y^{\prime}}$, the quartic (4) transforms to the cubic elliptic curve

$$
Y^{\prime 2}=X^{\prime 3}+\frac{\sum_{i=1}^{n} T_{i} U_{i}^{4}}{18} X^{\prime}
$$

Then if the elliptic curve (6) has positive rank (This is done by choosing appropriate rational values for $U_{i}$, and $T_{i}$.), by calculating $V, t, X, Y$ from the above relations and $X=U+t$, $Y=U-t$, and by some simplifications and canceling the denominators of $X=U+t, Y=$ $U-t, U_{i}$ and $T_{i}$, we may obtain infinitely many integer solutions for the DE (2). The proof is completed.

Remark 2.2. Generally, it is not essential for $Q$ to be square, the only important thing is the rank of the quartic elliptic curve (4) to be positive for getting infinitely many solutions for the DE (2). Note that if the constant number $Q$ is not square and the quartic (4) has positive rank, we may transform this quartic to a quartic elliptic curve with square constant number, and then use the Lemma 1.1 for transforming this new quartic to a cubic elliptic curve.

Now we work out some examples.

Example 1. $x^{4}+y^{4}=2 v^{4}+L w^{4}$ (given in the Introduction).

First of all, we solve the DE $x^{4}+y^{4}=2 v^{4}+L$.

Letting $x=v+t, y=v-t$, yields

$$
2 t^{4}+12 v^{2} t^{2}=L
$$

Then by taking $v t=z$, we get:

$$
z^{2}=\frac{-1}{6} t^{4}+\frac{L}{12}
$$

Then if $\frac{L}{12}$ is square (This means that $L=3 A^{2}$, where $A$ is a rational number.), we may transform the quartic (8) to a cubic elliptic curve.

Let $L=3 A^{2}$, then the quartic elliptic curve (8) becomes

$$
z^{2}=\frac{-1}{6} t^{4}+\frac{A^{2}}{4}
$$


With the inverse transformation $t=\frac{A X}{Y}$ and $z=\frac{-A}{2}+\frac{t^{2} X}{A}$, the corresponding cubic elliptic curve is

$$
Y^{2}=X^{3}+\frac{A^{2}}{6} X
$$

Then if the elliptic curve (10) has positive rank, we obtain infinitely many solutions for the DE $x^{4}+y^{4}=2 v^{4}+L w^{4}$, with $L=3 A^{2}$. Also if we let $A=2 A^{\prime}\left(L=12 A^{\prime 2}\right)$, we get

$$
x^{4}+y^{4}=2 v^{4}+12 A^{\prime 2} w^{4}=2 v^{4}+2\left(6 A^{\prime 2}\right) w^{4} .
$$

Thus we conclude that if the elliptic curve

$$
Y^{2}=X^{3}+\frac{2 A^{\prime 2}}{3} X
$$

has positive rank ( $A^{\prime}$ is a rational number.), we obtain infinitely many solutions for the DE

$$
x^{4}+y^{4}=2 v^{4}+12 A^{\prime 2} w^{4}=2 v^{4}+2\left(6 A^{\prime 2}\right) w^{4} .
$$

This is the same DE (1) given in the Introduction for $k=6 A^{\prime 2}$, that has been studied by some authors (see [1-6]). Then we may solve the DE (1) for all of the values $k=6 A^{\prime 2}$, which rank of the elliptic curve (11) is positive.

As an example, by letting $L=3$, the quartic (8) becomes

$$
z^{2}=\frac{-1}{6} t^{4}+\frac{1}{4}
$$

With the inverse transformation $t=\frac{X}{Y}$ and $z=\frac{-1}{2}+t^{2} X$, we get the elliptic curve $Y^{2}=X^{3}+\frac{1}{6} X$. Rank $=1$.

Generator: $P=(X, Y)=\left(\frac{25}{36}, \frac{145}{216}\right) . \quad(t, z)=\left(\frac{30}{29}, \frac{409}{1682}\right)$.

Solution: $2209^{4}+1391^{4}=2 .(409)^{4}+3 .(1740)^{4}$, i.e., the sums of two biquadratics equal to the sums of five biquadratics.

By taking $L=\frac{1}{3}$, the quartic (8) becomes

$$
z^{2}=\frac{-1}{6} t^{4}+\frac{1}{36}
$$

With the inverse transformation $t=\frac{\frac{1}{3} X}{Y}$ and $z=\frac{-1}{6}+\frac{t^{2} X}{\frac{1}{3}}$, this maps to the new elliptic curve $Y^{2}=X^{3}+\frac{1}{54} X$.

Rank $=1$.

Generator: $P=(X, Y)=\left(\frac{1}{36}, \frac{5}{216}\right) . \quad(t, z)=\left(\frac{2}{5}, \frac{-23}{150}\right)$.

Solution: $3 .(1)^{4}+3 .(47)^{4}=6 .(23)^{4}+60^{4}$, i.e., the sums of six biquadratics equal to the sums of seven biquadratics.

Letting $L=588\left(A=14\right.$ or $\left.A^{\prime}=7\right)$, in the quartic (8), yields

$$
z^{2}=\frac{-1}{6} t^{4}+49
$$


With the inverse transformation $t=\frac{14 X}{Y}$ and $z=-7+\frac{t^{2} X}{14}$, this maps to the cubic elliptic curve $Y^{2}=X^{3}+\frac{98}{3} X$.

Rank $=1$.

Generator: $P=(X, Y)=\left(\frac{7}{81}, \frac{1225}{729}\right) . \quad(t, z)=\left(\frac{18}{25}, \frac{-4373}{625}\right)$.

Solution: $4049^{4}+4697^{4}=2 .(4373)^{4}+588 .(450)^{4}$.

By taking $L=75$, the quartic (8) becomes

$$
z^{2}=\frac{-1}{6} t^{4}+\frac{25}{4}
$$

With the inverse transformation $t=\frac{5 X}{Y}$ and $z=\frac{-5}{2}+\frac{t^{2} X}{5}$, the cubic elliptic curve is $Y^{2}=$ $X^{3}+\frac{25}{6} X$.

Rank $=1$.

Generator: $P=(X, Y)=(24,118) . \quad(t, z)=\left(\frac{60}{59}, \frac{17155}{6962}\right)$.

Solution: $4871^{4}+1991^{4}=2 .(3431)^{4}+75 .(1416)^{4}$.

Also we have: $2 P=\left(X^{\prime}, Y^{\prime}\right)=\left(\frac{11771761}{2005056}, \frac{42760419191}{2839159296}\right)$.

By using this new point, we obtain another solution for the DE (for $L=75$ ) as

$$
\begin{aligned}
& x=357853717430881, \\
& y=114207083041439, \\
& v=121823317194721, \\
& w=121097507148912 .
\end{aligned}
$$

Finally if in (8) we let $L=2 .\left(6 r^{2}+1\right)$, where $r$ is a rational number, we get

$$
z^{2}=\frac{-1}{6} t^{4}+\frac{6 r^{2}+1}{6}
$$

Let $\frac{6 r^{2}+1}{6}=g^{2}$, then we obtain $r=\frac{1}{12 \alpha}-\frac{\alpha}{2}$ and $g=\frac{\alpha}{2}+\frac{1}{12 \alpha}$.

We have $L=2 .\left(6 r^{2}+1\right)=2 .\left(6 \cdot\left(\frac{1}{12 \alpha}-\frac{\alpha}{2}\right)^{2}+1\right)$, then also we get

$$
z^{2}=\frac{-1}{6} t^{4}+\left(\frac{\alpha}{2}+\frac{1}{12 \alpha}\right)^{2} .
$$

With the inverse transformation $t=\frac{2\left(\frac{\alpha}{2}+\frac{1}{12 \alpha}\right) X}{Y}$ and $z=-\left(\frac{\alpha}{2}+\frac{1}{12 \alpha}\right)+\frac{t^{2} X}{2\left(\frac{\alpha}{2}+\frac{1}{12 \alpha}\right)}$, the corresponding cubic elliptic curve is

$$
Y^{2}=X^{3}+\left(\frac{2}{3}\right) \cdot\left(\frac{\alpha}{2}+\frac{1}{12 \alpha}\right)^{2} X .
$$

Therefore we conclude that if the elliptic curve (19) has positive rank, we obtain infinitely many solutions for the $\mathrm{DE} x^{4}+y^{4}=2 v^{4}+L w^{4}$, with $L=2 \cdot\left(6 r^{2}+1\right)=2 .\left(6 \cdot\left(\frac{1}{12 \alpha}-\frac{\alpha}{2}\right)^{2}+1\right)=$ $\left(\frac{1}{3}\right) \cdot\left(\frac{1}{2 \alpha}+3 \alpha\right)^{2}$.

As an example, if in (19), we take $\alpha=1$, we get $L=\frac{49}{12}$ and $Y^{2}=X^{3}+\frac{49}{216} X$. 
Rank $=1$.

Generator: $P=(X, Y)=\left(\frac{7}{36}, \frac{49}{216}\right) . \quad(t, z)=\left(1, \frac{-5}{12}\right)$.

Solution: $12 .(7)^{4}+12 .(17)^{4}=24 \cdot(5)^{4}+49 \cdot(12)^{4}$.

Example 2. $x^{4}+y^{4}=2 u^{4}+2 v^{4}+L w^{4}$.

Firstly, we solve the DE $x^{4}+y^{4}=2 u^{4}+2 v^{4}+L$.

Again, letting $x=v+t, y=v-t$ yields

$$
2 t^{4}+12 v^{2} t^{2}=2 u^{4}+L
$$

Now by taking $v t=z$, we get:

$$
z^{2}=\frac{1}{6} u^{4}+\frac{L-2 t^{4}}{12} .
$$

Let us transform the quartic (21) to a cubic elliptic curve. Let

$$
q^{2}=\frac{L-2 t^{4}}{12}
$$

(Remark:* Again, to transform the quartic elliptic curve to a cubic elliptic curve, and to find appropriate values for $q, t$, with $L$ given, so that the elliptic curve (21) has positive rank, $\frac{L}{12}$ must be a square. Then with an arbitrary L given, we may obtain $q$, $t$, from the elliptic curve (22) if the rank of the quartic (21) or the coresponding cubic elliptic curve (23) is $\geq 1$, and consequently obtain infinitely many solutions for the above DE.*) curve

Now with the inverse transformation $u=\frac{2 q X}{Y}$ and $z=-q+\frac{u^{2} X}{2 q}$, we get the new elliptic

$$
Y^{2}=X^{3}+\frac{-L+2 t^{4}}{18} X
$$

Then if the above elliptic curve has positive rank (This is done by choosing appropriate values for $t$, such as rank of the above elliptic curve to be $\geq 1$, and $\frac{L-2 t^{4}}{12}$ to be square.), we obtain infinitely many solutions for the above DE.

As an example, if we take $t=3, q=1$, we get $L=174$. Then we obtain $z^{2}=\frac{1}{6} u^{4}+1$. With the inverse transformation $u=\frac{2 X}{Y}$ and $z=-1+\frac{u^{2} X}{2}$, we get the new elliptic curve $Y^{2}=X^{3}-\frac{2}{3} X$.

Rank $=1$.

Generator: $P=(X, Y)=\left(-\frac{2}{9}, \frac{10}{27}\right) . \quad(u, z)=\left(-\frac{6}{5},-\frac{29}{25}\right)$.

Solution: $196^{4}+254^{4}=2 \cdot\left(29^{4}+90^{4}\right)+174 \cdot(75)^{4}$.

This time, first we take $L=3$, and then obtain appropriate value for $t$, such as $\frac{3-2 t^{4}}{12}$ to be a square. (i.e., $L=3 g^{2}\left(\frac{L}{12}\right.$ must be a square.), where $g$ is a rational number.).

Let

$$
A^{2}=\frac{3-2 t^{4}}{12}=-\frac{t^{4}}{6}+\frac{1}{4}
$$


With the inverse transformation $t=\frac{X}{Y}$ and $A=\frac{-1}{2}+t^{2} X$, this maps to the new elliptic curve $Y^{2}=X^{3}+\frac{1}{6} X$.

Rank $=1$.

Generator: $P=(X, Y)=\left(\frac{25}{36}, \frac{145}{216}\right) . \quad(t, A)=\left(\frac{30}{29}, \frac{409}{1682}\right)$.

So by taking $t=\frac{30}{29}$ and $L=3$, in the main elliptic curve (21), we get:

$$
z^{2}=\frac{1}{6} u^{4}+\left(\frac{409}{1682}\right)^{2}
$$

With the inverse transformation $u=\frac{\frac{409}{841} X}{Y}$ and $z=\frac{-409}{1682}+\frac{u^{2} X}{\frac{409}{841}}$, the cubic elliptic curve is $Y^{2}=X^{3}-\frac{167281}{4243686} X$.

Rank $=2$.

One of Generators: $P_{1}=(X, Y)=\left(\frac{204919225}{1025024256}, \frac{343427056595}{32817176580096}\right) . \quad(u, z)=\left(\frac{15803760}{1701053}, \frac{203932086158809}{5787162617618}\right)$. Solution (for the DE $x^{4}+y^{4}=2 u^{4}+2 v^{4}+3 w^{4}$ ):

$$
\begin{aligned}
& x=12325316479952166913, \\
& y=197738927590609, \\
& u=11962044377817259513, \\
& v=55620069019200, \\
& w=5986719949260,
\end{aligned}
$$

i.e., the sums of two biquadratics equal to the sums of seven biquadratics.

Example 3. $x^{4}+y^{4}=2 u^{4}+2 v^{4}+L w^{4}+K r^{4}$.

At first, we solve the DE $x^{4}+y^{4}=2 u^{4}+2 v^{4}+L w^{4}+K$.

Letting $x=v+t, y=v-t$ yields

$$
2 t^{4}+12 v^{2} t^{2}=2 u^{4}+L w^{4}+K .
$$

Now, by taking $v t=z$, we get:

$$
z^{2}=\frac{1}{6} u^{4}+\frac{L w^{4}-2 t^{4}+K}{12} .
$$

Let us transform the quartic (27) to a cubic elliptic curve. Let

$$
q^{2}=\frac{L w^{4}-2 t^{4}+K}{12}
$$

(Remark:* Again, to transform the above quartic elliptic curve to a cubic elliptic curve and to find appropriate values for $w, t, q$, we need the rank of (27) to be $\geq 1$, as an example, we may write

$$
q^{2}=\frac{L w^{4}-2 t^{4}+K}{12}=\frac{L w^{4}}{12}+\frac{-2 t^{4}+K}{12},
$$

then $\frac{-2 t^{4}+K}{12}$ must be a square to transform the above quartic to a cubic elliptic curve. Let $B^{2}=$ $\frac{-2 t^{4}+K}{12}$. To find appropriate values for $B$, and $t$, we must have $K=3 G^{2}$. After getting the values for $B$, and $t$, we solve the quartic

$$
q^{2}=\frac{L w^{4}}{12}+\frac{-2 t^{4}+K}{12}=\frac{L w^{4}}{12}+B^{2}
$$


for $q$, and $w$ (with $L$ given and B obtained). Finally, after getting appropriate values for $q$, and $w$, we solve

$$
z^{2}=\frac{1}{6} u^{4}+\frac{L w^{4}-2 t^{4}+K}{12}=\frac{1}{6} u^{4}+q^{2}
$$

for $z$, and $u$ (With appropriate value of q just obtained.). If the above quartic or its coresponding cubic elliptic curve (29) has positive rank, we obtain infinitely many solutions for the above DE.*)

Then with the inverse transformation $u=\frac{2 q X}{Y}$ and $z=-q+\frac{u^{2} X}{2 q}$, we get the new elliptic curve

$$
Y^{2}=X^{3}+\frac{-L w^{4}+2 t^{4}-K}{18} X
$$

Then if the above elliptic curve has positive rank (This is done by choosing appropriate values for $w$, and $t$, so that the rank of the above elliptic curve is $\geq 1$, and making $\frac{L w^{4}-2 t^{4}+K}{12}$ be square.), we obtain infinitely many solutions for the above DE. Of course, we may first choose $w, t$, and the other parameters, then choose $L, K$ such as rank of the elliptic curve (29) to be $\geq 1$.

As an example, if we take $t=3, L=-1, w=5, q=3$, we get $K=895 \neq 3 G^{2}$. Then we obtain $z^{2}=\frac{1}{6} u^{4}+9$.

With the inverse transformation $u=\frac{6 X}{Y}$ and $z=-3+\frac{u^{2} X}{6}$, the cubic elliptic curve is $Y^{2}=$ $X^{3}-6 X$.

Rank $=1$.

Generator: $P=(X, Y)=(-2,2)$.

By using the point $2 P=\left(X^{\prime}, Y^{\prime}\right)=\left(\frac{25}{4}, \frac{-115}{8}\right)$, we get $(u, z)=\left(-\frac{60}{23}, \frac{2163}{529}\right)$.

Solution (for the point $2 P$ ): $2308^{4}+866^{4}+2645^{4}=2 .\left(1380^{4}\right)+2 .\left(721^{4}\right)+895 .(529)^{4}$.

This time, first we take $t=2, w=\frac{1}{2}$, and then obtain appropriate values for $L, K$, such as $\frac{L w^{4}-2 t^{4}+K}{12}=1$, and $L+K=-2$.

(We wish the number of quadratics in both sides of the above DE to be equal and then show how some numbers can be written as sums of some biquadrates in two different ways with different coefficients.)

Then we get: $L=\frac{-736}{15}$ and $K=\frac{706}{15}$. By plugging these values into (27), we obtain

$$
z^{2}=\frac{1}{6} u^{4}+1
$$

With the inverse transformation $u=\frac{2 X}{Y}$ and $z=-1+\frac{t^{2} X}{2}$, the above quadratic becomes $Y^{2}=X^{3}-\frac{2}{3} X$.

Rank $=1$.

Generator: $P=(X, Y)=\left(\frac{-2}{9}, \frac{10}{27}\right) . \quad(u, z)=\left(\frac{-6}{5}, \frac{-29}{25}\right)$.

Solution (for the DE $15 x^{4}+15 y^{4}+736 w^{4}=30 u^{4}+30 v^{4}+706 r^{4}$ ):

$$
\text { 15. }(71)^{4}+15 .(129)^{4}+736 \cdot(25)^{4}=30 \cdot(60)^{4}+30 \cdot(29)^{4}+706 \cdot(50)^{4} .
$$


Note that: $15+15+736=30+30+706$. Therefore, we may choose the appropriate values for the coefficients of the Diophantine equations and show how some numbers can be written as sums of some biquadrates in two different ways with different coefficients.

We used the Sage software for calculating the rank of the elliptic curves (see [7]).

\section{Acknowledgements}

We are very grateful to the unknown referees and Prof. Vassia Atanassova, Managing Editor, for careful reading of the paper and giving several useful comments which improved the quality of this paper. .

\section{References}

[1] Bremner, A., \& Choudhry, A., \& Ulas, M. (2014) Constructions of diagonal quartic and sextic surfaces with infinitely many rational points, International Journal of Number Theory, 10(7), 1675-1698.

[2] Bremner, A. (1987) On Euler's quartic surface, Math. Scand, 61, 165-180.

[3] Charmichael, R. D. (1915) Diophantine Analysis, John Wiley and Sons, Inc.

[4] Izadi, F., \& Nabardi, K. (2016) Diophantine equation $X^{4}+Y^{4}=2\left(U^{4}+V^{4}\right)$, Math. Slovaca, 66(3), 557-560.

[5] Janfada, A. S., \& Shabani-solt, H. (2015) On Diophantine equation $x^{4}+y^{4}=2 z^{4}+2 k w^{4}$, Far East Journal of Mathematical Sciences, 100(11), 1891-1899.

[6] Mordell, L. J. (1969) Diophantine Equations, Academic Press, Inc.

[7] Sage software, Available online: http://sagemath.org.

[8] Washington, L. C. (2008) Elliptic Curves: Number Theory and Cryptography, ChapmanHall. 